INTERNATIONAL JOURNAL OF

SYSTEMATIC BACTERIOLOGY

Vol. 19, No. 3 July 1959

pp. 263-265

Copyright 1969, Iowa State University Press

\title{
PROPOSAL OF A NEOTYPE STRAIN FOR MORAXELLA LACUNATA
}

\section{S. D. Henriksen}

Kaptein W. Wilhelmsen og Frues Bakteriologiske Institutt, University of Oslo, Rikshospitalet, Oslo, Norway

ABSTRACT. The strain Morax 260 (ATCC 17967 ) is proposed as the neotype strain for Moraxella lacunata. The strain NCTC 7911 isconsidered a satisfactory reference strain for the M. lacunata biotype liquefaciens.

Type strains have not yet been designated for the Moraxella species $M$. lacunata and $M$. liquefaciens. In a recent paper (Henriksen and $B \phi$ vre $1 \overline{968}$ ) it was proposed to unite the two species under the name $M$. lacunata. If this proposal is accepted, it would not be necessary to name a neotype strain for $M$. nonliquefaciens, but it may be useful to have a reference strain for the biotype, which this former species represents. The purpose of this paper is to propose a neotype strain for $M$. lacunata and a reference strain for the $\underline{M}$. lacunata biotype $\overline{\text { liquefaciens. }}$.

The neotype strain for $\underline{M}$. lacunata

Several strains have been considered. All of them appear to be old strains, and most of them show a marked tendency to roughness. This may be a disadvantage, but recent, smooth isolates appear to be difficult to obtain. Also, it may be preferable to choose a strain which has some standing in the Moraxella literature. For these reasons I propose the strain Morax 260. This is an old strain from the collection of Institute Pasteur in Paris. The exact origin of the strain is unknown to me, but it is stated to have been isolated from a case of conjunctivitis. The strain was included in a previous study (Henriksen 1952), and it has been included in a collection of strains now being studied by the Moraxella Subcommittee. It has recently been reexamined and found to have the following characters: 
Morphology: Medium-sized, Gram-negative, nonmotile, nonencapsulated, nonsporulating rods, arranged singly and in pairs with a marked tendency to pleomorphism, mainly in the form of production of chains and long filaments. The colonies on blood agar are delicate, flat, ca. $1 \mathrm{~mm}$ diameter after 24-48 h, distinctly rough.

Growth characteristics: Does not grow on plain media such as peptone water or Hugh and Leifson's OF-medium, but grows on media containing blood or body fluids. No growth at room temperature, but grows at $30-37^{\circ} \mathrm{C}$.

Biochemical reactions: Does not acidify sugar media. Liquefies coagulated serum and gelatin (if serum is added). Gives a positive catalase reaction and oxidase reaction both with dimethyl- and tetramethyl-p-phenylenediamine. Negative reactions for indol and $\mathrm{H}_{2} \mathrm{~S}$. Urease reaction negative. No deamination of phenylalanine. Nitrate is reduced. No growth in citrate medium or other simple mineral media. No haemolysis.

Antibiotic sensitivity: Strongly sensitive to penicillin and most of the other common antibiotics. Does not produce penicillinase.

According to these characters it appears to be a typical strain suitable as a neotype strain.

\section{A reference strain for the biotype liquefaciens}

Among the strains studied in this laboratory (B $\phi$ vre 1965), the strain NCTC 7911 (ATCC 17952) appears to be a typical strain of this biotype. It mostly appears as a comparatively plump rod, mostly arranged in pairs, and without any marked tendency to pleomorphism or roughness. Its characters are mostly the same as of the strain described above, but it is less fastidious and is more willing to grow on simple media, e.g. the OF-medium of Hugh and Leifson and nutrient agar and broth. The characters of the strain have been confirmed by recent reexamination.

It is proposed that this strain be used as a reference strain of the biotype liquefaciens, and that it may be considered a neotype strain of $\mathrm{M}$. liquefaciens by those who wish to retain this organism $\overline{a s}$ a separate species. 


\section{REF ERENCES}

B申vre, K. 1965. Studies on transformation in Moraxella and organisms assumed to be related to Moraxella. 5. Streptomycin resistance transformation between serumliquefying, nonhaemolytic moraxellae, Moraxella bovis and Moraxella nonliquefaciens. Acta path. et mic $\overline{r o b i o l}$. scandinav. 65: 435-449.

Henriksen, S. D. 1952. Moraxella: Classification and taxonomy. J. gen. Microbiol. 6:318-328. and K. B $\phi v r e, 1968$. The taxonomy of the genera Moraxella and Neisseria. J. gen. Microbiol. 51:387-392. 